\title{
Vulvar Angiomyofibroblastoma
}

National Cancer Institute

\section{Source}

National Cancer Institute. Vulvar Angiomyofibroblastoma. NCI Thesaurus. Code C45446.

An angiomyofibroblastoma arising from the vulva. 